\title{
LA GESTIÓN DE LAS PERSONAS EN EL NUEVO ENTORNO EMPRESARIAL DE LA COMUNICACIÓN
}

\section{THE MANAGEMENT OF PEOPLE IN THE NEW BUSINESS COMMUNICATION}

María José Pérez Serrano: Facultad de Ciencias de la Comunicación de la Universidad de San Jorge. Zaragoza (España)

mjperez@usj.es

María Romero Calmache: Facultad de Ciencias de la Comunicación de la Universidad de San Jorge. Zaragoza (España)

$\underline{\text { mromero@usj.es }}$

\section{CURRÍCULUM VITAE}

Licenciada en Periodismo, doctora en Ciencias de la Información por la Universidad Complutense de Madrid y MBA en la especialidad de Gestión Financiera. Su tesis doctoral ha sido merecedora del Premio Extraordinario de Doctorado del curso académico 2006-2007. Está acreditada por la ANECA como Profesor Ayudante Doctor, Profesor de Universidad Privada y Profesor Contratado Doctor. Ha pertenecido, entre otros, al cuerpo docente de las facultades de Comunicación de la Universidad San Jorge y de la Universidad Pontificia de Salamanca y del Instituto de Postgrado y Formación Continua de la Universidad Pontificia de Comillas (ICADE), así como del Departamento de Periodismo IV (Empresa Informativa) de la Facultad de Ciencias de la Información de la Universidad Complutense de Madrid, donde en estos momentos imparte docencia. Su trayectoria y producción científica se vincula al estudio de la Empresa Informativa y, específicamente, a la concentración de medios 
de comunicación y su influencia en el pluralismo. Es autora de comunicaciones y artículos; ha desarrollado estancias académicas en centros universitarios nacionales e internacionales, y forma parte de grupos de investigación

Licenciada en Comunicación. Profesora de Mix de Comunicación de la Facultad de Ciencias de la Comunicación de la Universidad San Jorge en Zaragoza (España).

\section{RESUMEN}

Este artículo constituye una reflexión que intenta conjugar varios aspectos, hasta ahora, tibiamente tratados en lo concerniente al mercado de la información. Como cualquier otra, la industria de la comunicación no es ajena a las circunstancias económicas que vivimos en esta primera década del siglo XXI y en este contexto, estas páginas estudian cómo la empresa de comunicación se relaciona con sus recursos humanos e indaga en cómo las personas, dentro del ámbito empresarial de la información, adquieren peso específico, sufren una importante transición laboral derivada del cambio en sus rutinas de trabajo y contribuyen a la permanencia de su unidad económica dentro de llamado cuarto sector económico. Específicamente, el trabajo se centra en estas relaciones (intraempresariales) y que cómo estos públicos internos, unas veces como protagonistas y otras como espectadores, asisten al quehacer diario de la actividad empresarial y contribuyen a transmitir una imagen singular (valor, formación, etc.) de la organización al exterior, lo que perfila, como imprescindibles, una planificación, dirección y gestión vanguardistas y específicas, más aún en estos tiempos de fluctuaciones constantes. 


\section{PALABRAS CLAVE}

Empresa de comunicación - Públicos Internos - Recursos Humanos - Management Stakeholders

\section{ABSTRACT}

This paper is a reflection which combines several aspects regarding the information market, which, up until now, has been dealt with cautiously. Like any other, the communication industry is not immune to the economic circumstances in which we live in this first decade of XXI century and, in this context, this article will study how media companies are related to human resources and it will explore how people, within the information business field, have gained more importance; they suffer an important transition that is a result of changes in their work routines, and which contribute to the permanence of its economic unity within the so-called fourth economic sector. Specifically, the paper focuses on these relationships (intracorporate) and how this internal public, sometimes as players and other times as spectators, attending the daily routine of business, help to transmit a singular image (values, training, etc.) outside the organization, which needs planning, management and specific, pioneering administration, more so in these times of constant fluctuations.

\section{KEY WORDS}

Media business - Internal Publics - Human Resources - Management - Stakeholders 


\section{ÍNDICE}

1. Estado de la cuestión

2. Límites metodológicos

3. Planteamiento teórico

4. Las relaciones de la empresa de comunicación con su capital humano

4.1 Internet como paradigma del nuevo entorno

4.2 La relación "mercantil" del individuo con la empresa de comunicación

4.3 La comunicación como base de la otra relación

5. A modo de cierre

6. Referencias bibliográficas

\section{TEXTO}

\section{Estado de la cuestión}

La actual empresa de comunicación ha desarrollado una importante carrera hacia el progreso a partir de un reconocible esfuerzo por investigar cada una de las áreas que le afectan, es decir, de considerar su realidad como un todo parcelado y estudiar, y mejorar, cada una de esas divisiones para conseguir un resultado cada vez más beneficioso tanto en términos económicos, como en cuanto a su posición en la sociedad que le circunda. En esta línea, los intangibles 1 ganan cada día protagonismo a medida que se van conociendo los correlatos positivos que posee su eficaz gestión. Con el fin de indagar en uno de esos aspectos se plantean estas 
páginas que comienzan estableciendo, como no podía ser de otro modo, los límites dentro de los cuales se van a desenvolver los contenidos.

No se encuentra entre nuestros cometidos observar cuáles son las relaciones sinérgicas de la organización empresarial con todos sus stakeholders 2; ni siquiera con todos los correspondientes al ámbito interno (accionistas, trabajadores, clientes y proveedores); tampoco lo es analizar las normas básicas de la comunicación empresarial, sino estudiar cómo la empresa de comunicación se relaciona con sus recursos humanos y ver cuáles son las consecuencias de la fundamental mutación tecnológica en las rutinas de trabajo de los profesionales de la empresa que tiene como objeto social la información. Asimismo, se pretende concretar cómo, también en el contexto mediático, se perfila como imprescindible una planificación y gestión específicas y contemporáneas, que fijen como reto la adaptación a estas nuevas coordenadas que se producen en el mundo actual, donde las tecnologías vinculadas a la red condicionan el ecosistema social.

1- En términos genéricos, por intangibles estratégicos se entiende la "imagen corporativa", "la marca", "la cultura corporativa y gestión del conocimiento", "la reputación corporativa" y la "responsabilidad social corporativa" principalmente. Según el proyecto MERITUM (Measuring Intangibles to Understand and Improve Innovation Management), "por intangibles se entiende los recursos intangibles e inversiones y actividades intangibles" (Chaminade, 2001).

2- "Públicos de interés para la empresa" (Carrillo et al., 2008: 10). Según Boatright (1993) "la teoría de los stakeholders no es mas que un marco de referencia para ayudarnos a poner en marcha la función verdaderamente difícil: decidir exactamente 
qué obligaciones de las empresas caen bajo la denominación de responsabilidad social de las empresas".

volver al primer apartado volver al principio del artículo volver al principio

\section{Límites metodológicos}

Mas lo dicho anteriormente quedaría sujeto a la nomenclatura de grandes objetivos, necesarios, pero no suficientes, en un trabajo con signos de cientificidad. Por eso, es imprescindible partir de una declaración conjetural, una proposición tentativa acerca de las relaciones entre dos o más fenómenos o variables, que podría quedar formulada de la siguiente manera: debido, entre otras razones, a la incursión de la red, las relaciones de la empresa de comunicación con su capital humano, en los últimos años, divergen en dos sentidos: el primero es que Internet ha modificado la tarea de los profesionales; el segundo es que ha variado la forma en que la organización se vincula y relaciona con ellos.

Situados ya en este plano analítico, se aplicará, el método científico; se incidirá en el hipotético-deductivo para la pertinente claridad y rigor intelectual, y se completará con el análisis de los parámetros que sustentan la investigación y sus conclusiones.

\section{Planteamiento teórico}

Con cierta asiduidad se vinculan las relaciones públicas con la imagen que proyecta la empresa al exterior. Precisamente y para enfatizar en el plano interior, el planteamiento novedoso de este artículo se sustenta en dos coordenadas conjugadas en una simbiosis que las complementa y une. Esos dos ejes de reflexión son, por un lado, que la empresa objeto de estudio es aquella que tiene como fin, precisamente, la comunicación; y, por otro, que se analizan las relaciones intraempresariales que esa 
organización posee como ente con personalidad jurídica. Específicamente, el trabajo se centra en las vinculaciones con las personas que forman parte de esa empresa y que, unas veces como protagonistas y otras como espectadores, asisten al quehacer diario de la actividad empresarial.

En este sentido, es clave entender la triple estructura conceptual de la que partimos: la definición de empresa de comunicación como unidad económica inserta en una economía deslocalizada y globalizada; el prisma peculiar de las relaciones públicas, y, lo más importante, la formulación de un concepto de recursos humanos fundado en la persona.

Para definir el primero de estos conceptos nos apoyaremos en la propuesta de García Jiménez (1998: 7) que señala que "la empresa es un órgano de la sociedad que tiene por objetivo primario obtener beneficios y lo logra gracias a la conjugación de tres elementos básicos: una organización, la combinación de una serie de factores productivos y la existencia del mercado". En las últimas décadas se ha observado la ineficacia de un modelo funcional rígido en el que cada individuo integrante tenía una función y un papel específico, lo que ha llevado a diversos riesgos que se han solucionado adoptando nuevos modelos donde existe la coordinación e integración de cada una de las partes en un todo común, y donde cualquier acción o decisión implica e influye en el resto de departamentos. El cambio ha determinado la aparición de la llamada organización transfuncional, con diferentes subsistemas, en la cual desempeña un papel decisivo la comunicación interna (García, 1998).

Si hablamos de la empresa de comunicación, habría que añadir que su objeto social es la información en sentido amplio y que su resultado es la plasmación de esa idea empresarial que se lleva a cabo a través de la puesta en marcha de una estructura compleja que aglutina a lo que Iglesias denomina "factores productivos elementales" 
y "factores dispositivos". Si los recursos humanos pertenecen, sin ninguna duda, a los primeros, es el paraguas organizacional que los ampara (perteneciente a los segundos) el que sirve para que, en una empresa (también de comunicación), el todo sea mayor que la suma de sus partes.

En este punto, debemos acotar el segundo de los términos. Durante años, las Relaciones Públicas se han desarrollado teniendo como base prácticas poco rigurosas hasta llegar a ser consideradas como una reconocida función de gestión y dirección. En este sentido, Cutlip y Center (1952) las definen como "el esfuerzo planeado para influenciar la opinión mediante el buen carácter y la ejecución responsable, basados en una comunicación de dos direcciones mutuamente satisfactorias". En la actualidad, los modernos planteamientos de las RRPP tienden hacia el modelo simétrico bidireccional (Two-way symmetrical model), propugnado, desde un punto de vista teórico, por Grunig y Hunt (2003). Este paradigma establece, partiendo de la investigación, una mediación entre la organización y el resto de sistemas del entorno para ajustar las políticas empresariales al público interno y externo al que se dirige.

El tercero de los conceptos, el de recursos humanos, lo definimos, simplemente, como "las personas que trabajan en la organización. Este término se ha ido aceptando de forma general durante la última década porque expresa la creencia de que los trabajadores constituyen un recurso valioso, y, a veces, irremplazable, para la empresa" (Gómez-Mejía et al., 2002: 2). Dicho de otra forma, las personas forman las organizaciones, desempeñan una labor retribuida, pero, además de su trabajo, de ellas se espera, siempre, algo más casi siempre asociado con el componente de personalidad y de motivación que tienen los individuos y que impregnan, incluso, los objetivos de la empresa. 
Siguiendo con esta línea de reflexión, en los últimos tiempos se ha estudiado el concepto de "hombre complejo" que presupone que "en sus intercambios con el ambiente empresarial, los individuos están motivados por un deseo de utilizar sus habilidades para solucionar los problemas que afrontan. En el sistema particular de la personalidad individual, el patrón de valores, percepciones y motivaciones es el resultado de la interacción de las características biológicas del individuo con la experiencia que acumula en su crecimiento" (Chiavenato, 2000). El estudio de las motivaciones del personal, las relaciones y la comunicación con éste, ha suscitado numerosas investigaciones que dan como fruto unas pautas teóricas para lograr un flujo eficaz de información en la empresa, tanto descendente como ascendente. El objetivo principal es la simbiosis entre los objetivos organizacionales e individuales para lograr alcanzarlos, aprehenderlos conjuntamente. Para ello, según Barnard (1971: 281), es necesario fomentar la participación de la persona en la organización.

\section{Las relaciones de la empresa de comunicación con su capital humano}

Si hubiese que enunciar de una manera sencilla, casi telegráfica, en qué consisten estas relaciones y en qué ejes se desenvuelven, habría que responder que las segundas son las de eficacia y eficiencia, y que las relaciones son, fundamentalmente, de dos tipos: una mercantil y otra que denominamos comunicacional; aunque éstas, como hemos señalado, han tenido, en los últimos tiempos, un elemento impulsor en la dinámica diaria, que no es otro que la incursión de Internet.

\subsection{Internet como paradigma del nuevo entorno}

Esta transformación, que podría quedar circunscrita a un medio y no a lo esencial, implica la adaptación imprescindible de las unidades económicas que se desenvuelven en un mercado, el informativo, cada vez más competitivo y que 
encuentra uno de sus mayores desafíos en hacer frente a una competencia feroz, tanto en el plano local como en el supranacional.

En ellas, lo digital ha supuesto el descubrimiento de un horizonte nuevo no solamente en la distribución de contenidos, sino también en la estructura, estrategias $\mathrm{y}$ en el proceso productivo de todas las organizaciones y empresas. En general, y siguiendo a Gómez-Mejía (2001, 5-6) podemos señalar que el impacto de Internet en los recursos humanos recae sobre diversos aspectos, entre los que podemos destacar: * Necesidad de una mayor capacidad de redacción: las empresas han descubierto que la tecnología de Internet genera una fuerte demanda de trabajadores que puedan gestionar eficazmente los mensajes de correo electrónico. Esta cualidad es clave si las empresas quieren mantener la lealtad de los inquietos clientes por Internet, y reducir, así, la probabilidad de que se vayan a la competencia con un simple toque de teclado.

* Nueva definición del puesto de trabajo: Internet está planteando una pregunta compleja en torno a qué constituye la actividad principal de una empresa $u$ organización, es decir, si se trata de un conjunto de productos relacionados, una combinación de conocimiento y propiedad intelectual, o de un proceso para proporcionar servicios. El resultado es que los puestos de trabajo son cada vez más ambiguos y el viejo paradigma de adecuar las capacidades de las personas a los puestos de trabajo se está quedando obsoleto en muchas organizaciones. En la era de Internet, la capacidad de adaptación se ha convertido en una característica clave necesaria para tener éxito.

* Posibilidad de tener una dirección general centrada en los recursos humanos: Internet permite a las empresas tratar los detalles operativos de recursos humanos con mucha más rapidez y eficacia. 
* Y otros aspectos a considerar podrían ser la ruptura de las barreras del mercado de trabajo, la dificultad de conservar a los mejores trabajadores y el aprendizaje por Internet.

\subsection{La relación "mercantil" del individuo con la empresa de comunicación}

Como se ha indicado, junto con los económicos, financieros y técnicos, los recursos humanos constituyen la realidad del tejido empresarial; mas, si en todos ellos el paso del tiempo ha dejado su huella y motivado su evolución, en éstos ha modificado incluso su nomenclatura, adaptándose, en unos casos, a una eufemística forma de entender y comunicar la realidad, $\mathrm{y}$, en otros, siendo reflejo real de un cambio en el espíritu empresarial.

Convengamos, como fórmula de trabajo, que el término "dirección de personal" pertenece a un pasado obsoleto y que, como las modernas prácticas de gestión y management casi siempre han llegado con cierto retraso a las empresas de comunicación, ese planteamiento ya no pertenece a la realidad más cotidiana. Y, a partir de ahí, intentemos perfilar la evolución a la que hace referencia el epígrafe.

Desde que los trabajadores adquiriesen unas condiciones dignas, la evolución en este asunto ha ido asociada a un paulatino perfeccionamiento de las situaciones laborales y a un interés creciente por el aprendizaje, la especialización, la responsabilidad, la realización, el desarrollo y el reconocimiento del empleado. El cambio actual, pues, incide sobre la concepción del valor que tienen los trabajadores para la empresa con base en las ciencias del comportamiento. En esta conceptualización, se abandona la idea de que "la persona es un recurso, es decir, un elemento que provee de fuerza de trabajo a la empresa tal, para pasar a la consideración de la persona como un elemento que posee recursos, es decir, conocimientos, capacidades y actitudes" 
(Albizu, 2006: 51). Así, queda anclada en el pasado la idea "contable" del gasto en selección, capacitación y motivación y se asume la consideración de todos estos elementos como inversión (Claver et al., 2006).

Asimismo, debemos indicar que en el sintagma capital humano se incorporan tres conceptos importantes: los recursos humanos, ya que en algunos de los puestos se hace alusión a la interacción entre el empleado y la organización (psicología de los recursos humanos); los procesos organizacionales, dado que se incide en macroprocesos donde lo importante es el comportamiento colectivo de las personas en relación con la estructura socio-técnica (psicología de las organizaciones), y el trabajo (psicología del trabajo), puesto que los trabajadores, con sus comportamientos y dificultades, son verdaderos protagonistas activos (Boada i Grau, 2001).

Cuando hablamos de la relación mercantil, entendemos por esta la que comienza incluso con la planificación de los recursos humanos y que adquiere carta de legalidad con el proceso de contratación. Indudablemente, asimilamos el término "mercantil" en sentido laxo y no se circunscribe estrictamente al tipo de contrato que se le hace al trabajador. Ni que decir tiene que la relación se hace patente a través de dicho contrato, es decir, mediante el libre consentimiento de las partes y en el cual el individuo aporta su fuerza de trabajo y la empresa le remunera la acción. Pero, lo que aquí denominamos relación "mercantil" está vinculado, como se apunta desde el principio, con las funciones de planificación, administrativas (políticas de retribución, por ejemplo), técnicas (formación, desarrollo integral, evaluación del rendimiento) innovadoras y productivas 3 que lleva a cabo el responsable de recursos humanos siguiendo las indicaciones y directrices del empresario. Esta relación tiene un carácter burocrático y se conecta con las ventas, el volumen de producción, el número de clientes, la rentabilidad, etc. 
Uniendo ambos elementos, en el campo comunicativo, hemos pasado -o deberíamos dejar atrás- la idea de una mano de obra "periodística" para insertar a las corporaciones en un nuevo modelo basado en las personas en el que, sin perder de vista su objetivo empresarial de maximizar beneficios, éstas también las consideren un factor primordial; que encuentren el equilibrio entre el capital humano en el ámbito de la instrumentalización del ser humano y la visión personalista que enarboló su dignidad, y que, frente a la visión económico-utilitarista moderna, busque una dimensión humanista del trabajo (Galindo, 2001) en aras a la obtención de dos metas: la mejora continua y, progresivamente, la calidad total, y, así, hacer real la máxima de Ortega y Gasset (1983), la vida humana es quehacer y el quehacer ético es quehacerse.

3- Para Jac Fitz-enz, la función de recursos humanos tiene que basarse sobre el objetivo de añadir valor mediante la eficacia en los procesos que le competen. La Dirección de Recursos Humanos (DRH) tiene como función principal crear y comunicar, en primera instancia a los otros directores y en segundo término a los empleados, los medios que les permitan poner a funcionar su imaginación para producir en todo momento acciones positivas, que se van a traducir en valor añadido. Esta es la función de productividad y, por ello, se puede afirmar que a la DRH le competen todas las áreas porque ella es el motor para hacerlas más productivas (Claver, 2003).

\subsection{La comunicación como base de la otra relación}

En 2004, Stephen Robbins señalaba que el $70 \%$ de nuestras horas de vigilia es comunicación. Un año más tarde, María Ángeles Durán (2005) indicaba que las personas dedican 860 horas anuales a trabajar. Sin ningún afán exhaustivo, si se 
hallara ese porcentaje sobre el tiempo dedicado a la actividad laboral encontraríamos que 602 horas al año nos pasamos en el trabajo "comunicándonos". Una pincelada curiosa que apoya la importancia que tiene esta relación de la que pretendemos esbozar ahora sus rasgos principales.

Desde un punto de vista de estructural y de gestión, en los últimos tiempos, la comunicación está irrumpiendo con fuerza como instrumento de los nuevos estilos de dirección empresarial (Sánchez Calero, 2005), fomentando unos valores de claridad y apertura, en lo que Borrini (1997) denomina la "empresa transparente". Esa dirección es de la que la American Association for the Advancement of Science afirma que "por muy buena que sea nuestra investigación del futuro, en última instancia, nunca podremos evitar el dilema de que todo nuestro conocimiento lo es del pasado; y que todas nuestras decisiones pretenden "entender" el futuro."

Para que la comunicación sea eficaz debe haber un foco común del que emane una línea informativa coherente que tenga su reflejo sustancial -y, tal vez, diversificadotanto en los públicos externos como internos; es decir, se trata de ofrecer un único mensaje, pero adaptado a cada uno de los públicos. Y respecto a los segundos, son los empleados los que, por convivir diariamente en el seno de la realidad organizacional, no deben ver descuidado su nivel de comprensión de la misma y que éste sea, además de intuitivo y participante, estandarizado, razonado y oficial, máxime aún teniendo en cuenta que "el mayor capital de las empresas en el siglo XXI está dado por su capital humano" (Pedrotti, 2007).

En este contexto, y de una manera casi natural, surgen tres términos que sirven de sustento a la exposición: por un lado, las relaciones con los públicos internos basadas en la comunicación 4; por otro, la responsabilidad social de la empresa, en este caso de comunicación; por último, la ética, estrictamente vinculada a la anterior. Los tres, 
unidos a otros aspectos, forjan la "imagen de la empresa, síntesis de su identidad". Por tanto, siguiendo a Fanjul (2008), "no se puede construir una sin conocer bien la otra. La imagen debe expresar los aspectos más favorables, destacables y diferenciadores que posea en esencia la organización, ya que una imagen que no esté basada en la realidad corporativa, tarde o temprano se verá descubierta y, cuando esto ocurra, la pérdida de credibilidad será más fuerte que el trabajo de imagen desarrollado hasta el momento". Respecto a las relaciones con los públicos internos, conviene señalar que, en términos absolutos, todavía no se le da la importancia debida 5; pero, aún así, se habla de la comunicación que los tiene como protagonistas, como recurso o herramienta indispensable. En esta línea, García Jiménez (1998) afirma que "la comunicación interna es la energía del sistema, sólo definible por sus efectos. Su expresión sensible es la organización, que dota a la empresa de equilibrio y estabilidad mediante la realimentación positiva y negativa. Tiene multiplicidad de fines, funciones y objetivos". Se dice de ella que la aplican como un factor clave para la rentabilidad y un aliado para conseguir sus objetivos estratégicos en situaciones de cambio. Andreu Pinillos (1996) asegura que esta comunicación ayuda en aspectos fundamentales de la competitividad empresarial porque transmite su cultura, su misión, su visión, sus valores, sus mensajes, sus objetivos generales, sus principales noticias, e implica a la plantilla en los aspectos esenciales del negocio. Y, además, crea relaciones eficientes entre los distintos públicos, grupos o equipos de las empresas, logrando así aproximarse a uno de los retos de la comunicación interna que es la "generación de valor" colaborando en "la cadena de valor" de las compañías (Morales, 2008).

Por supuesto que no nos referimos exclusivamente a la comunicación unidireccional que emana de la cúpula del staff organizativo y llega en forma de cascada a los estamentos inferiores, sino una comunicación integral, formal, informal, ascendente, descendente, horizontal, en red, etc. 
Los trabajadores prefieren que sus relaciones con sus superiores se basen en una comunicación satisfactoria, bilateral, cara a cara y personal. Y, para ello, la alta dirección debe hacer un esfuerzo por compartir información con los empleados; por reforzar la comunicación con mensajes escritos para evitar la distorsión del significado; por expresar mensajes en palabras llanas; por contar exactamente la verdad en los límites que esta pueda ser emitida; por dar la información oportuna; por repetir y por ofrecer la información en pequeñas dosis, y por utilizar, si es necesario, métodos audiovisuales que refuercen la comunicación oral (López, 2008).

Para ayudar en estos cometidos, la incorporación de Internet en la actividad cotidiana de cualquier empresa ha sido esencial. De hecho, se puede decir que la red de redes ha hecho que también las relaciones públicas de las que hablamos varíen de forma sustancial, y, en ellas, el empleo del "correo electrónico es, sin duda, la principal táctica ya que supone la transmisión de mensajes a destinatarios seleccionados mediante técnicas telemáticas y cuyas ventajas resultan evidentes: rapidez, interactividad, multidifusión, facilidad de fijación al destinatario, confidencialidad, disminución del gasto en papel y otros insumos" (Rojo, 2002).

En cuanto a la segunda, algunos expertos afirman que la Responsabilidad Social Corporativa (RSC) “debe comenzar por casa. Sólo se podrá obtener un verdadero compromiso de nuestros empleados, en el momento que éstos se sientan plenamente identificados con la realidad empresarial. Las comunicaciones internas bien gestionadas promueven la cooperación, la unión y la coexistencia, dentro de una realidad sujeta a condicionantes e identificadores institucionales; cobrando relevancia en la gestión de tareas y el reconocimiento de las realidades de cada individuo y de su grupo de trabajo" (Pedrotti, 2007). En realidad, la RSC promueve una nueva ética -tercer elemento- en los negocios (Salas, 2007: 69) ya que redunda en 
las sinergias que hay entre el propio eje empresarial (idea y desarrollo del dueño) y los colectivos que con él se relacionan. Y, en esta línea, "las empresas han visto en la relación economía-ética un beneficio inequívoco para sus intereses y para sus objetivos estrictamente empresariales" (Cortés, 2006: 48).

Por lo tanto, si todos estos aspectos contribuyen al diseño de una imagen empresarial favorable y que ésta ayuda, en última instancia, a fidelizar a los cliente $y$, subsidiariamente, a aumentar el nivel de producción, podemos decir que las relaciones comunicacionales con el capital humano que conforma el ente jurídico cuyo objeto social es la información, fundadas en una actitud ética para con ellos, deben centrar la atención de los directivos, ya que, si no lo hacen así no podrán entrar en el restringido club que define Tom Peters como empresas excelentes; es decir, aquellas que convergen hacia la ética (Ramos, 2001); poseen una reconocida preocupación por la "calidad" en todos sus ámbitos de gestión, y resultan ser las pioneras en la aplicación de la comunicación interna como una herramienta de gestión y un signo exterior de modernidad (Morales, 2008).

4- Las relaciones públicas y la gestión del conocimiento tienen un vínculo importante dentro de la organización. La primera puede usarse como herramienta de la segunda (La Fe, 2008).

5- Más del $40 \%$ de los encuestados por DIRCOM afirman que no existen o desconocen la existencia en su organización de estudios para conocer la opinión y las expectativas de los empleados sobre su situación profesional (Román, 2004).

\section{A modo de cierre}


Es cierto que, una vez superados los requerimientos básicos tales como un salario y unas condiciones dignas de trabajo, la gradación de las relaciones que hemos establecido varía dependiendo del tipo de empresa de que se trate y, muchas veces, también de los recursos económicos que se destinen a estos menesteres.

La relación mercantil se supedita a las funciones de la Dirección de Recursos Humanos y esas funciones pueden ceñirse, como ha sido costumbre, a la gestión de altas y bajas, o llegar a la socialización del trabajador; sin embargo, la existencia e intensidad de las relaciones comunicacionales muestra un avance sustancial en la tipología de organización: si se llevan a cabo, contribuyen a que aumente el sentido de pertenencia de trabajador; si no se fomentan, éste se considerará simplemente un colaborador, alejado de la política y los objetivos empresariales. Por ello, la comunicación interna es el pilar básico para que una organización sea eficaz; es fundamental que la alta dirección esté dispuesta a compartir información y que se produzca un contacto personal entre directivos y subordinados. El capital intelectual y el goodwill, también desde un punto de vista contable, proporcionan valor y rendimiento a la institución. Y los capitales humano y estructural conforman el capital intelectual, definido de forma simple (Edvinssons et al, 2003). Pero, sea como sea, la fortaleza competitiva de la empresa radica por igual en los tangibles e intangibles de la organización y las sinergias que de ellos surgen.

En las empresas de comunicación (como pueden ser aquellas que tienen por objeto la producción de un medio impreso o una televisión) los rasgos fundamentales de una gestión y dirección científicas siempre han llegado con cierto retraso. Todas presentan elementos de estabilidad, que le hacen tener una estructura propia y resistente, y también elementos que fluctúan con el paso del tiempo. Pero a la luz de la disección realizada podemos decir que dentro de la investigación de la 
comunicación en las organizaciones, las más importantes empresas de comunicación hacen esfuerzos considerables por situarse entre las más vanguardistas en cuanto a lo que a gestión de la comunicación se refiere.

Incluso así, el nuevo panorama empresarial impone un planteamiento coherente y exige una adaptación realista a los cambios. En todo ello, las relaciones con los públicos internos resultan ser una de las variables decisivas de esta capacidad de evolución y, que, sin duda, tienen un impacto efectivo sobre las decisiones estratégicas de la empresa.

Como síntesis conclusiva, podemos decir que Internet (paradigma del nuevo entorno de la comunicación) ha modificado las condiciones de trabajo, las tareas y la estructura de recursos humanos -las personas- de la tradicional empresa informativa, y que esta realidad exige una nueva forma de entender las relaciones "públicas" que mantienen los managers con su capital humano en el entorno mediático.

\section{Referencias bibliográficas}

ALBIZU, Eneka: Cambios en la función de recursos humanos. En: ALBIZU GALLASTEGI, Eneka; LANDETA RODRÍGUEZ, Jon (coord.): Dirección estratégica de los recursos humanos. Teoría y práctica. 2006. Madrid. Editorial: Pirámide. Pág. 51-62.

ANDREU, Alberto: Comunicación interna, un paseo por el tiempo. En: Harvard Deusto Business Review, nº 70, enero-febrero. Bilbao. 1996. Editorial: Deusto.

BARNARD, Chester I.: As funções do executive. São Paulo. 1971. Editorial: Atlas. 
BOADA I GRAU, Joan (dir.): Capital humano, 52 casos prácticos. Manual de supuestos prácticos sobre recursos humanos y procesos organizacionales. Barcelona. 2001. Editorial: Granica, Col. Management

BOATRIGHT, J.: Ethics and the conduct of business. Engle-wood Cliffs, NJ. 1993. Editorial: Prentice-Hall.

BORRINI, Alberto: La empresa transparente. Buenos Aires. 1997. Editorial: Atlántida.

CARRILLO, María Victoria; TATO, Juan Luis: La comunicación empresarial del siglo XXI. La gestión de los activos intangibles. En: Lecciones del Portal, Portal de la Comunicación, Institut de la Comunicació UAB, www.portalcomunicacion.com. 2008.

CHAMINADE, Cristina: La sociedad del conocimiento y su impacto en la empresa: medición y gestión de los intangibles. En: Revista Madri+d, Tribuna del Debate, núm. $3, \quad$ marzo 2001. http://www.madrimasd.org/revista/revista3/tribuna/tribunas3.asp. Madrid, 2001.

CHIAVENATO, Idalberto: Administración de Recursos Humanos. Santafé de Bogotá. 2000. Editorial: McGraw Hill, $5^{\circ}$ Ed.

CLAVER, Enrique; GASCÓ, José Luis; LLOPIS, Juan: Los recursos humanos en la empresa: un enfoque directivo. Madrid. 2003. Editorial: Civitas Economía y Empresa Tratados y manuales, $2^{\mathrm{a}}$ ed. 
CORTÉS GARCÍA, Francisco Joaquín: La ética empresarial desde la perspectiva de los costes de transacción. Un enfoque teórico. En: Boletín Económico de ICE, núm. 2899, 11-17 de diciembre de 2006.

CUTLIP, Scott M.; CENTER, Allen H.: Effective public relations. Englewood Clips. 1952. Editorial: Prentice-Hall.

DUELO, Ignacio: ¿Cómo se comunica el cambio al público interno? En: ¿Podemos hablar? Reflexiones y aconteceres de la comunicación. http:// podemoshablar.blogspot.com/2008/03/cmo-se-comunica-el-cambio-alpblico.html. Argentina. 2008.

DURÁN, María Ángeles: El uso del tiempo en la vida cotidiana. Madrid. 2005. Editorial: CSIC.

EDVINSSONS, Leif; MALONE, Michael S.: El Capital Intelectual. Barcelona. 2003. Editorial: Gestión 2000.com.

FANJUL, Carlos: La importancia de la comunicación no verbal en la configuración de la imagen corporativa. En: Icono 14, julio 2008. Editorial: Asociación Científica de Investigación de las NNTT de la Educación.

GALINDO GARCÍA, A.: Capital humano y trabajo. En: FLECHA ANDRÉS, J.R.: Marketing y recursos humanos, Salamanca. 2001. Editorial: Servicio Publicaciones Universidad Pontificia de Salamanca.

GARCÍA JIMÉNEZ, Jesús: La Comunicación Interna. Madrid. 1998. Editorial: Díaz de Santos. 
GÓMEZ MEJÍA, Luis R.; BALKIN, David B.; CARDY, Robert L.: Dirección y gestión de recursos humanos. Madrid. 2001. Editorial: Prentice Hall, $3^{\mathrm{a}}$ ed.

GRUNIG, James E. y HUNT, Todd: Dirección de Relaciones Públicas. Barcelona. 2003. Editorial: Gestión 2000.com.

LA FE JIMENEZ, Raúl Yoel: Las relaciones públicas y la gestión del conocimiento. En: Contribuciones a la Economía. Enero 2008. http://www.eumed.net/ce/2008a/ryfj.htm.

LÓPEZ FRAILE, Luis Antonio: Cómo comunicarse eficazmente con los empleados. 2008.

http://www.microsoft.com/spain/empresas/rrhh/comunicar_empleados.mspx.

MORALES SERRANO, Francisca: La comunicación interna. Herramienta estratégica de gestión para las empresas. En: Red DirCom Iberoamericana. 2008. http:/ / www.reddircom.org/textos/f-serrano.pdf.

ORTEGA Y GASSET, José: Obras completas. Madrid. 1983. Editorial: Alianza Editorial.

PEDROTTI, Guillermo José: “RPI: Relaciones Públicas Internas”, 27 de septiembre de 2007, Público Meta, El Blog de las Relaciones Públicas para las Relaciones Públicas, http://publicometa.fullblog.com.ar/post/rpi_relaciones_publicas_internas_2811908 99763. 
RAMOS HIDALGO, Encarnación: La ética del marketing: importancia de la ética y responsabilidad social para la efectividad organizacional. 2001. Caja San Fernando, Sevilla.

ROBBINS, Stephen P.: Comportamiento organizacional. 2004. México. Editorial: Pearson-Educación, $10^{\mathrm{a}}$ ed.

ROJO VILLADA, Pedro Antonio: ¿Viejas estrategias para nuevos canales?: El escenario para unas relaciones públicas globales y ubicuas. En: Razón y Palabra, núm. 25. 2002. México, febrero-marzo 2002.

ROMÁN, Yolanda (coord.): Expectativas, prácticas y resultados de la comunicación interna en empresas e instituciones españolas. Estudio de opinión informada en organizaciones líderes en comunicación interna. Asociación de Directivos de Comunicación (DIRCOM). 2004. Madrid.

SALAS FUMÁS, Vicente: Economía del gobierno empresarial orientado a los interesados: El caso de la empresa intensiva en conocimiento. En: Economiaz, núm. 65, $2^{\circ}$ cuatrimestre 2007. Pág. 69.

SÁNCHEZ CALERO, María Luisa: Desarrollo de la comunicación externa en la empresa. En: Sala de Prensa, núm. 83, Año VII, Vol. 3, septiembre 2005. 